\title{
Use of Body Mass Index to Predict the Prognosis of Patients with Remnant Gastric Cancer
}

\author{
Tomoyuki Matsunaga,*† Hiroaki Saito,* Tomohiro Osaki,* Yusuke Kono,* Yuki Murakami,* Hirohiko Kuroda,* \\ Yoji Fukumoto* and Yoshiyuki Fujiwara* \\ *Division of Surgical Oncology, Department of Surgery, School of Medicine, Tottori University Faculty of Medicine, Yonago \\ 683-8504, Japan
}

\begin{abstract}
Background Remnant gastric cancer (RGC) is an uncommon form of gastric cancer. The aim of this study was to investigate factors influencing the prognosis of patients with RGC.
\end{abstract}

Methods A total of 49 patients diagnosed with RGC and 214 patients with primary upper third gastric cancer (PUGC) at our institution between January 1990 and December 2014 were included. The clinicopathological characteristics, prognosis, and factors influencing prognosis were compared.

Results The body mass index (BMI) of RGC was significantly lower than that for PUGC $(P<0.0001)$. Multivariate analysis revealed that BMI and the depth of tumor invasion were independent prognostic factors in RGC. ROC analysis indicated that an optimal cut-off value for BMI was 20.6. Based on this value, patients were divided into two groups: $\mathrm{BMI}^{\mathrm{High}}(\geq 20.6)$ and $\mathrm{BMI}^{\mathrm{Low}}(<20.6)$. The 5-year survival rates of patients with $\mathrm{BMI}{ }^{\mathrm{High}}$ early gastric cancer, BMI ${ }^{\mathrm{High}}$ advanced gastric cancer, BMI ${ }^{\mathrm{Low}}$ early gastric cancer, and $\mathrm{BMI}^{\mathrm{Low}}$ advanced gastric cancer were 90\%, 83.3\%, 64.3\% and $33.8 \%$, respectively, and the difference was statistically significant $(P=0.00023)$.

Conclusion Our retrospective study indicated a poor prognosis of RGC compared with PUGC, and that BMI could predict the prognosis of RGC. The prognosis of patients with BMI ${ }^{\mathrm{Low}}$ advanced RGC was extremely poor.

Key words body mass index; gastric cancer; prognosis; remnant gastric cancer

Gastric cancer is one of the most common malignancies and death from gastric cancer ranks second among all cancer deaths worldwide. ${ }^{1}$ Remnant gastric cancer (RGC) is a less prevalent form of the disease, and the incidence of RGC has been reported as $1-7 \%$ of all gastric cancer cases. $^{2-4}$ The most frequently used definition of RGC is cancer in the remnant stomach after surgery for benign disease or cancer after surgery for gastric cancer found at least 5 years after the primary surgery. Surgery, in- cluding gastrectomy, has been the standard treatment for peptic ulcer disease in previous decades. ${ }^{5}$ Recent advances in peptic ulcer therapy, including specific treatment for Helicobacter pylori infection, have decreased the prevalence of affected individuals for whom gastrectomy is indicated. Therefore, it is likely that the incidence of RGC following gastrectomy for benign disease will decrease in the future. However, the incidence of RGC following gastrectomy for gastric cancer is increasing as a result of improved survival and early detection by mass screening, especially in several Asian countries. ${ }^{6}$ Therefore, rates of RGC following gastric cancer surgery have been increasing overall. ${ }^{7,8}$

RGC is often diagnosed at an advanced stage, with higher rates of invasion of adjacent organs and lymph node metastases, ${ }^{9,} 10$ low rate of curative resection and, consequently, a poor prognosis. ${ }^{10,11}$ It has been also reported that the prognosis for RGC is significantly worse than that for primary upper third gastric cancer (PUGC) that originates in the almost same lesion as RGC, when compared at the same stage, indicating the possibility that the characteristics of RGC might be different from those of primary gastric cancer.

Determining the postoperative prognosis in RGC patients is extremely important. Many studies have indicated that depth of invasion and presence or absence of lymph node metastasis are the most important prognostic factors in gastric cancer. ${ }^{12,13}$ However, the factors influencing the poor prognosis of patients with RGC remain unclear. The purpose of this study was to investigate the clinicopathological characteristics and outcomes

\footnotetext{
$†$ Present address: Department of Digestive Surgery, Osaka International Cancer Institute, Osaka 541-8567, Japan Corresponding author: Hiroaki Saito, $\mathrm{MD}, \mathrm{PhD}$ sai10@med.tottori-u.ac.jp

Received 2017 March 27

Accepted 2017 April 24

Abbreviations: ACTS-GC, Adjuvant Chemotherapy Trial of TS-1 for Gastric Cancer; BMI, body mass index; NRS2002, nutritional risk scoring 2002; OS, overall survival; PNI, Onodera's prognostic nutritional index; PUGC, primary upper third gastric cancer; RGC, remnant gastric cancer; SE, tumor penetration of serosa; SGA, Subjective Global Assessment; SI, tumor invasion of adjacent structure
} 
of surgery for RGC following distal gastrectomy. In particular, we examined the factors related to the prognosis of patients with RGC.

\section{MATERIALS AND METHODS Patients}

This study was based on a retrospective analysis of 49 RGC patients following distal gastrectomy who underwent curative gastrectomy at our institution between January 1990 and December 2014. During the same period, 214 patients were pathologically diagnosed with adenocarcinoma confined to the upper third of the stomach and were also included in the current study. The clinicopathological findings were determined according to the Japanese Classification of Gastric Carcinoma. ${ }^{14}$ Patients were periodically checked for early recurrence by diagnostic imaging (chest X-ray, double-contrast barium meal study, upper gastrointestinal fiberscopy, ultrasonography, and computed tomography). Causes of death and patterns of recurrence were determined by reviewing medical records, including laboratory data, ultrasonography, computed tomography, scintigrams, peritoneal punctures, and laparotomies, or by direct inquiry to family members. In some cases, postmortems were undertaken to determine the cause of death. Institutional review board approval was obtained (1607A064), and informed consent requirements were waived for this study.

\section{Statistical analysis}

The Mann-Whitney $U$ test and chi-square test were employed to evaluate differences in continuous and categorical variables, respectively. The Youden index was calculated using receiver operating characteristic (ROC) analysis to determine an optimal cutoff value for body mass index (BMI) for survival analysis. Survival curves were calculated using the Kaplan-Meier method. Differences between survival curves were examined with the log-rank test. Survival data shown in the current study were for overall survival (OS). We used multivariate analysis of factors considered prognostic of OS, with Cox's proportional hazards model. SPSS Statistics (ver. 24.0 software, IBM) was used for all statistical analyses, with $P<0.05$ considered statistically significant (2-tailed).

\section{RESULTS}

\section{Clinicopathological features of RGC and PUGC}

The clinicopathological characteristics of both RGC and PUGC are shown in Table 1. Patients with RGC were significantly older than those with PUGC $(P=0.002) .6$ patients with RGC were older than 80 years old (12.2\%) and one patient was over 85 years old. The frequency of male gender was significantly greater in RGC patients than in PUGC patients $(P=0.041)$. Furthermore, BMI was significantly lower in RGC patients than in PUGC patients $(P<0.0001)$. However, no significant differences were observed in tumor size, depth of tumor invasion, lymph node metastasis, blood vessel invasion, lymphatic vessel invasion, and state of disease between RGC and PUGC.

\begin{tabular}{|c|c|c|c|}
\hline & $\operatorname{RGC}(n=49)$ & PUGC $(n=214)$ & $P$ value \\
\hline Age (years) & $72.4 \pm 6.8$ & $66.2 \pm 12.2$ & 0.002 \\
\hline Gender & & & 0.041 \\
\hline Male & $43(87.8 \%)$ & $158(73.8 \%)$ & \\
\hline Female & $6(12.2 \%)$ & $56(26.2 \%)$ & \\
\hline Tumor size (mm) & $42.9 \pm 26.1$ & $36.3 \pm 23.1$ & 0.14 \\
\hline BMI & $20.3 \pm 2.6$ & $22.6 \pm 3.1$ & $<0.0001$ \\
\hline Histology* & & & 0.53 \\
\hline Differentiated & $25(51.0 \%)$ & $121(56.5 \%)$ & \\
\hline Undifferenciated & $24(49.0 \%)$ & $93(43.5 \%)$ & \\
\hline Depth of invasion $\dagger$ & & & 0.27 \\
\hline $\mathrm{T} 1$ & $23(46.9 \%)$ & $126(58.9 \%)$ & \\
\hline $\mathrm{T} 2$ & $6(12.2 \%)$ & $31(14.5 \%)$ & \\
\hline T3 & $9(18.4 \%)$ & $27(12.6 \%)$ & \\
\hline $\mathrm{T} 4$ & $11(22.5 \%)$ & $30(14.0 \%)$ & \\
\hline Lymph node metastasis & & & 0.44 \\
\hline Present & $13(26.5 \%)$ & $576.6 \%)$ & \\
\hline Absent & $26(73.5 \%)$ & $157(73.4 \%)$ & \\
\hline Lymphatic invasion & & & 0.53 \\
\hline Present & $28(57.1 \%)$ & $111(51.9 \%)$ & \\
\hline Absent & $21(42.9 \%)$ & $103(48.1 \%)$ & \\
\hline Blood vessel invasion & & & 0.20 \\
\hline Present & $27(55.1 \%)$ & $94(43.9 \%)$ & \\
\hline Absent & $22(44.9 \%)$ & $120(56.1 \%)$ & \\
\hline Stage of disease & & & 0.41 \\
\hline I & $26(53.1 \%)$ & $139(65.0 \%)$ & \\
\hline II & $13(26.5 \%)$ & $39(18.2 \%)$ & \\
\hline III & $10(20.4 \%)$ & $35(16.4 \%)$ & \\
\hline IV & $0(0 \%)$ & $1(0.4 \%)$ & \\
\hline
\end{tabular}

*Differentiated, papillary or tubular adenocarcinoma; undifferentiated, poorly differentiated, mucinous adenocarcinoma, and signet-ring cell carcinoma

$\dagger \mathrm{T} 1$, tumor has invaded lamina propria or submucosa; T2, tumor has invaded the muscularis propria; T3, tumor has invaded the subserosa; T4, tumor invasion is contiguous to or exposed beyond the serosa or tumor invades adjacent structures.

BMI, body mass index; PUGC, primary upper third gastric cancer; RGC, remnant gastric cancer.

\section{Prognosis of patients with RGC or PUGC}

The 5-year survival rates of patients with RGC and PUGC were $57.9 \%$ and $76.2 \%$, respectively, and the difference was statistically significant $(P=0.0094$, Fig. 1). In detail, no significant difference in prognosis was 


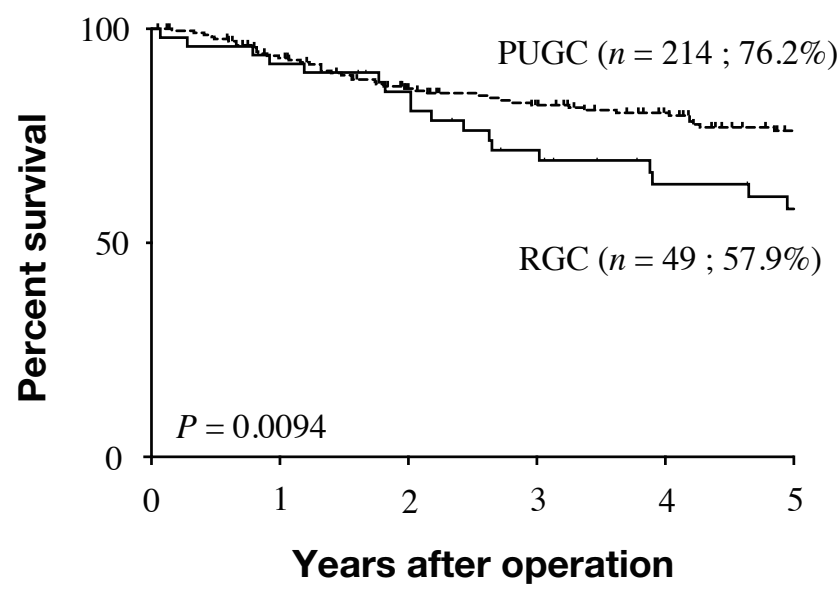

A

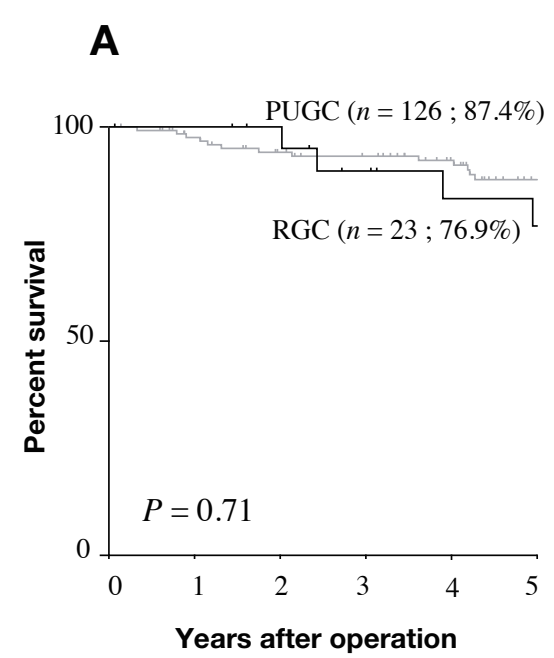

B

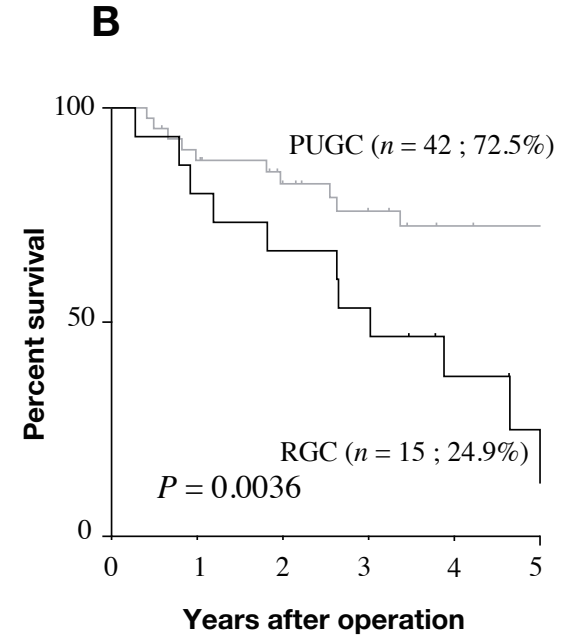

Fig. 1. Survival curves following gastrectomy of RGC and PUGC. The 5-year survival rates of RGC and PUGC were $57.9 \%$ and $76.2 \%$, respectively, and the difference was statistically significant $(P=0.0094)$. PUGC, primary upper third gastric cancer; RGC, remnant gastric cancer.

Fig. 2. (A) Survival curves of patients with either early RGC or early PUGC. (B) Survival curves of patients with either node-negative advanced RGC or node-negative advanced RGC. (C) Survival curves of patients with either node-positive advanced RGC or node-positive advanced RGC. PUGC, primary upper third gastric cancer; RGC, remnant gastric cancer.

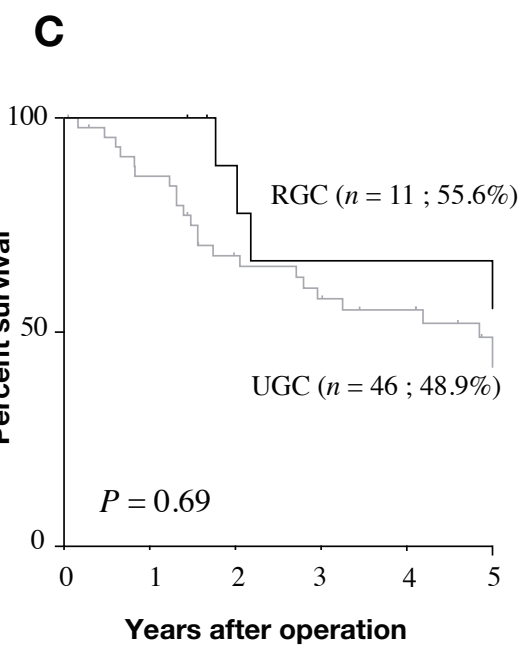

observed between early RGC patients and early PUGC patients $(P=0.71$, Fig. 2a). However, the prognosis of patients with node-negative advanced RGC was significantly worse than that of patients with node-negative advanced PUGC ( $P=0.0036$, Fig. $2 b)$. There was no significant difference in prognosis between node-positive RGC patients and node-positive PUGC patients $(P=$ 0.69 , Fig. 2c). Since a significant difference in prognosis was observed between node-negative advanced RGC and node-negative advanced PUGC, the sites of recurrence of either RGC or PUGC were determined in those patients. Peritoneal, hematogenous, and lymph node recurrence were observed in 3 (20\%), 3 (20\%), and 2 patients $(13.3 \%)$ with node-negative advanced RGC and $1(2.3 \%), 2(4.7 \%)$, and no patients $(0 \%)$ in node-negative advanced PUGC. Peritoneal recurrence was observed significantly more in RGC than in PUGC patients $(P=$ $0.049)$, although there was no significant difference in hematogenous $(P=0.1)$ and lymph node recurrence $(P=$ $0.064)$ between RGC and PUGC.

\section{Factors related to prognosis in RGC and PUGC}

We next examined factors related to prognosis in both RGC and PUGC. Univariate analysis revealed that BMI, tumor size, and depth of tumor invasion were significant prognostic factors in RGC, and that age, tumor size, depth of tumor invasion, lymph node metastasis, lymphatic invasion, and venous invasion were significant prognostic factors in PUGC (Tables 2 and 3). Furthermore, multivariate analysis revealed that BMI and depth of tumor invasion were independent prognostic factors in RGC (Table 2). However, age, depth of tumor invasion, and lymph node metastasis were found to be independent prognostic factors in PUGC (Table 3).

Because BMI and depth of tumor invasion were independent prognostic indicators, we determined whether an accurate prognosis of RGC could be predicted using $\mathrm{BMI}$ and depth of tumor invasion. ROC analysis indicated that an optimal cut-off value for BMI was 20.6 (AUC $0.746, P=0.0031$ ) (Fig. 3). Based on this value, patients were divided into two groups as follows: $\mathrm{BMI}^{\mathrm{High}}(\geq$ 20.6) and $\mathrm{BMI}^{\mathrm{Low}}(<20.6)$. The 5-year overall survival 
Table 2. Univariate and multivariate analyses of factors prognostic of overall survival in patients with RGC

\begin{tabular}{|c|c|c|c|c|c|c|}
\hline & \multicolumn{3}{|c|}{ Univariate analysis } & \multicolumn{3}{|c|}{ Mutivariate analysis } \\
\hline & HR & $95 \% \mathrm{CI}$ & $P$ value & HR & $95 \% \mathrm{CI}$ & $P$ value \\
\hline Age* & 0.997 & $0.936-1.063$ & 0.936 & & & \\
\hline Gender (Male vs Female) & 0.776 & $0.180-3.345$ & 0.733 & & & \\
\hline BMI* & 0.723 & $0.579-0.902$ & 0.00417 & 0.731 & $0.563-0.949$ & 0.018 \\
\hline Histology (Differentiated vs Undifferentiated) $\dagger$ & 0.516 & $0.216-1.232$ & 0.136 & & & \\
\hline Tumor size* & 1.014 & $1.003-1.025$ & 0.01 & 0.994 & $0.973-1.016$ & 0.512 \\
\hline Depth of tumor invasion $(\mathrm{T} 1-\mathrm{T} 4) \ddagger$ & 1.837 & $1.289-2.616$ & 0.000755 & 1.989 & $1.122-3.527$ & 0.004 \\
\hline Lymph node metastasis (N0-N3)§ & 1.759 & $0.735-4.208$ & 0.205 & & & \\
\hline Lymphatic invasion (Ly0-Ly3)\| & 1.086 & $0.698-1.688$ & 0.715 & & & \\
\hline Venous invasion $(\mathrm{V} 0-\mathrm{V} 3) \mathrm{II}$ & 1.438 & $1.038-1.992$ & 0.029 & 0.994 & $0.598-1.509$ & 0.6 \\
\hline
\end{tabular}

*Continuous variable

†Histology: Differentiated, papillary or tubular adenocarcinoma; undifferentiated, poorly differentiated or mucinous adenocarcinoma, or signet ring cell carcinoma

$\$ \mathrm{~T} 1$, tumor has invaded lamina propria or submucosa; T2, tumor has invaded the muscularis propria; T3, tumor has invaded the subserosa; T4, tumor invasion is contiguous to or exposed beyond the serosa or tumor invades adjacent structures.

$\S \mathrm{N} 0$, no regional lymph node metastases; N1, metastasis in 1-2 regional lymph nodes; N2, metastasis in 3-6 regional lymph nodes; $\mathrm{N} 3$, metastasis in 7 or more regional lymph nodes.

|lLymphatic vessel invasion: ly0-ly3, grade of lymphatic invasion.

I[Blood vessel invasion: $\mathrm{v} 0$-v3, grade of venous invasion.

BMI, body mass index; CI, confidence interval; HR, hazard ratio; RGC, remnant gastric cancer.

\section{Table 3. Univariate and multivariate analyses of factors prognostic of overall survival in patients with PUGC}

\begin{tabular}{|c|c|c|c|c|c|c|}
\hline & \multicolumn{3}{|c|}{ Univariate analysis } & \multicolumn{3}{|c|}{ Mutivariate analysis } \\
\hline & HR & $95 \% \mathrm{CI}$ & $P$ value & $\mathrm{HR}$ & $95 \% \mathrm{CI}$ & $P$ value \\
\hline Age* & 1.074 & $1.045-1.104$ & $<0.0001$ & 1.078 & $1.047-1.110$ & 0.0001 \\
\hline Gender (Male vs Female) & 0.882 & $0.472-1.648$ & 0.694 & & & \\
\hline BMI* $^{*}$ & 0.986 & $0.913-1.065$ & 0.72 & & & \\
\hline Histology (Differentiated vs Poorly differentiated) & 1.148 & $0.669-1.969$ & 0.882 & & & \\
\hline Tumor size* & 1.013 & $1.004-1.023$ & 0.00479 & 0.998 & $0.983-1.013$ & 0.811 \\
\hline Depth of tumor invasion (T1-T4) & 1.682 & $1.367-2.070$ & $<0.0001$ & 1.399 & $1.002-1.955$ & 0.048 \\
\hline Lymph node metastasis (N0-N3) & 1.913 & $1.536-2.383$ & $<0.0001$ & 1.679 & $1.212-2.324$ & 0.0018 \\
\hline Lymphatic invasion (Ly0-Ly3) & 1.517 & $1.226-1.878$ & 0.00013 & 0.988 & $0.714-1.389$ & 0.981 \\
\hline Venous invasion (V0-V3) & 1.57 & $1.226-2.009$ & 0.00034 & 0.977 & $0.711-1.341$ & 0.885 \\
\hline
\end{tabular}

*Continuous variable

See Table 2 for details of histology, depths of invasion, lymph node metastasis, lymphatic invasion, and venous invasion.

BMI, body mass index; CI, confidence interval; HR, hazard ratio; PUGC, primary upper third gastric cancer.

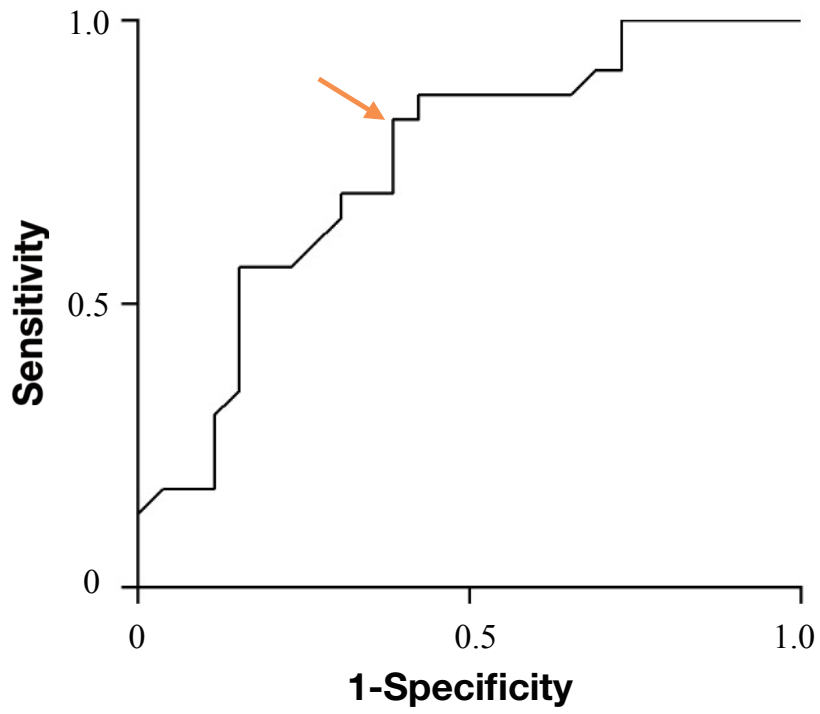

Fig. 3. ROC analysis indicated that an optimal cut-off value for BMI was 20.6. The BMI had good diagnostic accuracy (AUC 0.746 , sensitivity $82.6 \%$, specificity $61.0 \%, P=0.0031$ ). The arrow shows the optimal cutoff point. ROC, receiver operating characteristic.

rates of patients with $\mathrm{BMI}^{\mathrm{High}}$ early gastric cancer, BMIHigh advanced gastric cancer, BMI ${ }^{\text {Low }}$ early gastric cancer, and $\mathrm{BMI}^{\mathrm{Low}}$ advanced gastric cancer were 90\%, 83.3\%, $64.3 \%$ and $33.8 \%$, respectively, and the difference was statistically significant (Fig. 4a). The 5-year disease specific survival rates of patients with $\mathrm{BMI}^{\mathrm{High}}$ early gastric cancer, $\mathrm{BMI}^{\mathrm{High}}$ advanced gastric cancer, $\mathrm{BMI}^{\mathrm{Low}}$ early gastric cancer, and $\mathrm{BMI}^{\mathrm{Low}}$ advanced gastric cancer were 

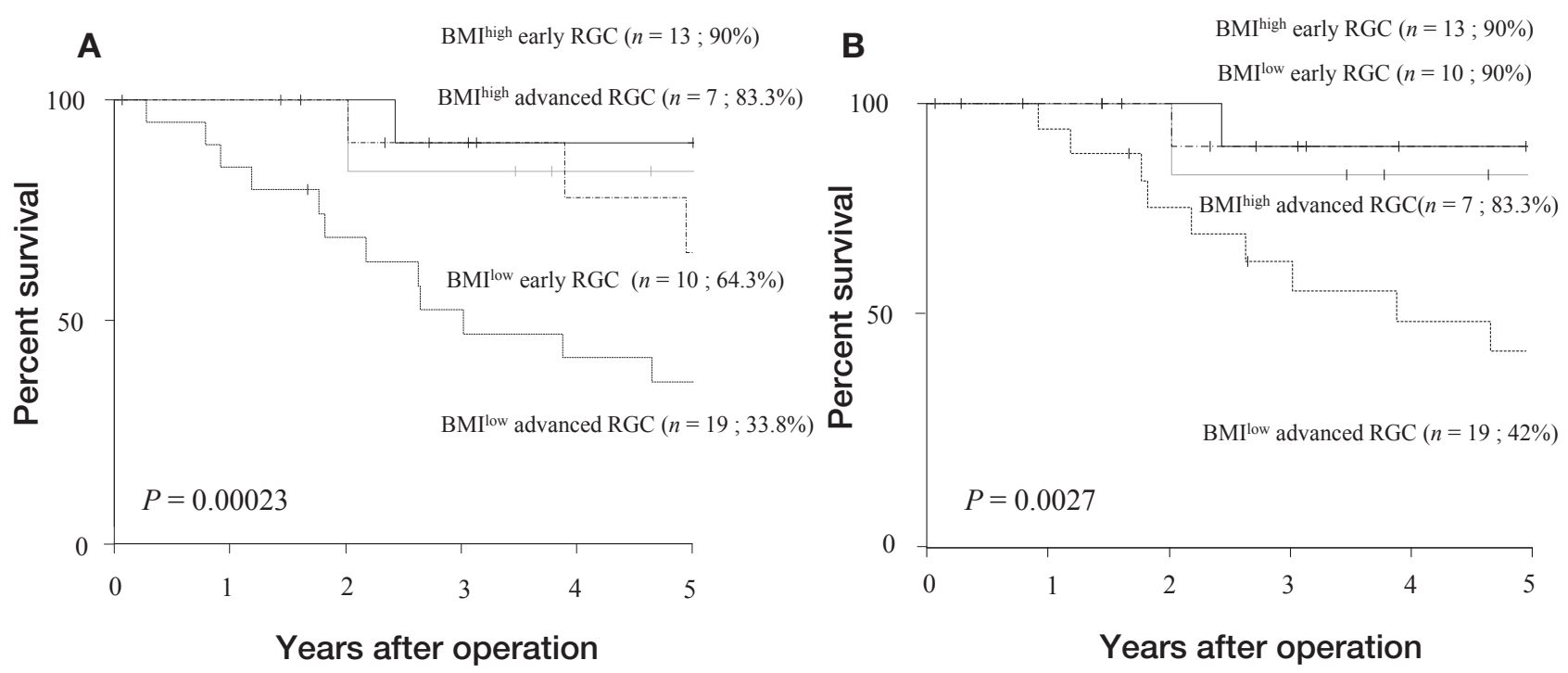

Fig. 4. Survival curves of patients with RGC according to BMI and depth of invasion. (A) The 5-year overall survival rates of patients with RGC. (B) The 5-year disease specific survival rates of patients with RGC. BMI, body mass index; RGC, remnant gastric cancer.

$90 \%, 83.3 \%, 90 \%$ and $42 \%$, respectively, and the difference was statistically significant (Fig. 4b). Furthermore, recurrence was observed in 11 patients $(57.9 \%)$ out of 19 with $\mathrm{BMI}^{\mathrm{Low}}$ advanced gastric cancer and 3 patients out of the remaining patients $(10 \%)$, a difference that was statistically significant $(P=0.0007)$. Two patients with RGC died of other cancer and 6 patients with RGC died of other disease such as pneumonia or heart failure or myocardial infarction in RGC.

\section{DISCUSSION}

We have demonstrated in the current study that the prognosis of RGC was significantly worse than that of PUGC. This result is similar to that previous reports. ${ }^{15,16}$ The poor prognosis of RGC may be affected by the stage distribution of the patients. In the study by Tokunaga et al., $43.1 \%$ of the RGC group comprised tumor penetration of serosa (SE) or tumor invasion of adjacent structure (SI) patients; in contrast, only $18.9 \%$ of the primary gastric cancer group included these patients. ${ }^{15}$ However, this does not seem to explain the poor prognosis observed in the current study because there was no significant difference in depth of invasion, lymph node metastasis, and stage of disease between RGC and PUGC patients. Another possibility is the molecular changes observed in RGC. The remnant stomach is frequently exposed to bile and pancreatic juice, which can induce molecular changes in the gastric mucosa. In a retrospective study with 130 patients, Leivonen et al. analyzed the cell proliferation rate in biopsy specimens from gastric remnants by immunohistochemical staining of Ki-67.17 The Ki-67 labeling index of tumors was significantly higher in the remnant stomach group, and is known to be associated with bile reflux and reconstruction without bile reflux. Baba et al. found that p53 overexpression in tumors was almost twice as common in patients with gastric cancer of the remnant stomach than in patients that had undergone distal gastrectomy for benign disease. ${ }^{18}$ It has also been demonstrated that p53 overexpression and higher Ki-67 labeling index are closely related to poor prognosis in gastric cancer. ${ }^{19}, 20$ Therefore, the poor prognosis observed for RGC patients in the current study might be related to molecular changes. Further investigations are therefore required to determine the correlation between poor prognosis and molecular changes in RGC.

Anatomical alterations and intraabdominal adhesions after initial surgery, as well as frequent combined resection of other organs, present difficulties for RGC surgery. Many studies have described an altered lymphatic pathway for RGC resulting from initial surgery. Furthermore, optimal lymph node dissection remains unclear thus far. It is likely that these surgical limitations are associated with poor prognosis in RGC.

It has recently been reported that preoperative nutritional status is a useful predictor of prognosis of gastric cancer patients. Several assessment tools can be applied to nutritional evaluation, such as Subjective Global Assessment (SGA), ${ }^{21}$ mini-nutritional assessment, ${ }^{22}$ nutritional risk scoring 2002 (NRS2002), ${ }^{23}$ and Onodera's prognostic nutritional index (PNI). ${ }^{24}$ Nozoe et al. have recently reported that PNI could predict the prognosis and biological aggressiveness of gastric cancer. ${ }^{25}$ With 
regard to BMI, it has been reported that underweight patients with $\mathrm{BMI}<18.5 \mathrm{~kg} / \mathrm{m}^{2}$ and low preoperative albumin levels had a significantly decreased OS after gastrectomy for cancer, ${ }^{26}$ indicating the close correlation between low BMI and poor prognosis in gastric cancer patients. It is likely that an initial gastrectomy places patients in a state of malnutrition. In the current study, BMI was significantly lower in patients with RGC than in those with PUGC. Furthermore, multivariate analysis indicated that BMI was an independent prognostic indicator, suggesting the usefulness of BMI to predict the prognosis of RGC patients.

Lymph node metastasis is one of the most important prognostic factors for patients who have undergone curative resection for gastric cancer. ${ }^{27,}{ }^{28}$ Lymph node metastasis was shown in the current study to be an independent prognostic indicator for PUGC. However, no significant difference in prognosis was observed between patients with lymph node metastasis and those without lymph node metastasis in RGC. Furthermore, lymph node metastasis was not an independent prognostic indicator for RGC in the current study. One possible explanation for this is that most lymph nodes are dissected in RGC patients whose initial operation was performed for gastric cancer, thus lowering the significance of lymph node metastasis as a prognostic indicator. Another possible explanation is insufficient lymph node dissection as a result of postoperative adhesion induced by an initial operation, which renders subsequent lymph node dissection difficult. Insufficient lymph node dissection is related to stage migration and might represent one reason why a significant difference in prognosis between RGC and PUGC was observed in node-negative but not node-positive advanced cancer.

The staging of gastric cancer according to the Japanese Classification of Gastric Cancer system is based on the depth of tumor invasion, status of lymph node metastasis, and presence of distant metastasis. In general, this staging system is used for RGC, although it is unclear how suitable it is for this disease subset. In this regard, our results indicated that factors reflecting the patient's nutritional status should be included in the staging system of RGC.

The Japanese Gastric Cancer Treatment Guideline recommends adjuvant chemotherapy with $\mathrm{S}-1$ for a year in stage II/III gastric cancer patients who have undergone gastrectomy with D2 lymph node dissection based on the results of the Adjuvant Chemotherapy Trial of TS-1 for Gastric Cancer (ACTS-GC). ${ }^{29}$ It remains unclear whether $\mathrm{S}-1$ is also effective in improving the prognosis of RGC. Since the prognosis of RGC is worse than that of PUGC, adjuvant chemotherapy might be necessary to improve prognosis in RGC patients. Based on our results, adjuvant chemotherapy should be administered to patients with BMI ${ }^{\text {Low }}$ advanced RGC, given their extremely poor prognosis. Furthermore, randomized control trials are required to determine effective adjuvant chemotherapy strategies in patients with RGC.

This retrospective study has several limitations. One major limitation is the small sample size. Furthermore, we included patients with RGC following distal gastrectomy for both benign diseases and gastric cancer, which might have influenced the clinical outcome. In this regard, it has been demonstrated that there was no significant difference in survival between RGC where the initial operation was performed for benign disease and that where the initial operation was performed for gastric cancer. ${ }^{15}$ In addition, the retrospective nature of our study could be considered another significant limitation, which may have influenced the results. Therefore, our findings should be confirmed by prospective studies.

In conclusion, our retrospective study indicated that the prognosis of RGC was poor compared with that of PUGC, and that BMI is useful to predict the prognosis of RGC. The prognosis of patients with BMI ${ }^{\text {Low }}$ advanced RGC was extremely poor, and recurrence rates among this subset of patients was extremely high. Therefore, multidisciplinary therapy, including preoperative chemotherapy and intensive adjuvant chemotherapy, might be necessary for patients with BMI ${ }^{\mathrm{Low}}$ advanced RGC.

The authors declare no conflict of interest.

\section{REFERENCES}

1 Jemal A, Bray F, Center MM, Ferlay J, Ward E, Forman D. Global cancer statistics. CA Cancer J Clin. 2011;61:69-90. PMID: 21296855.

2 Ahn HS, Kim JW, Yoo MW, Park DJ, Lee HJ, Lee KU, et al. Clinicopathological features and surgical outcomes of patients with remnant gastric cancer after a distal gastrectomy. Ann Surg Oncol. 2008;15:1632-9. PMID: 18379851.

3 Nozaki I, Nasu J, Kubo Y, Tanada M, Nishimura R, Kurita A. Risk factors for metachronous gastric cancer in the remnant stomach after early cancer surgery. World J Surg. 2010;34:1548-54. PMID: 20217411.

4 Sinning C, Schaefer N, Standop J, Hirner A, Wolff M. Gastric stump carcinoma - epidemiology and current concepts in pathogenesis and treatment. Eur J Surg Oncol. 2007;33:133-9. PMID: 17071041.

5 Svanes C, Salvesen H, Stangeland L, Svanes K, Soreide O. Perforated peptic ulcer over 56 years. Time trends in patients and disease characteristics. Gut. 1993;34:1666-71. PMID: 8282252

6 Maruyama K, Kaminishi M, Hayashi K, Isobe Y, Honda I, Katai H, et al. Gastric cancer treated in 1991 in Japan: data analysis of nationwide registry. Gastric Cancer. 2006;9:51-66. PMID: 16767357.

7 Ohashi M, Katai H, Fukagawa T, Gotoda T, Sano T, Sasako M. 
Cancer of the gastric stump following distal gastrectomy for cancer. Br J Surg. 2007;94:92-5. PMID: 17054314.

8 Fujita T, Gotohda N, Takahashi S, Nakagohri T, Konishi M, Kinoshita T. Relationship between the histological type of initial lesions and the risk for the development of remnant gastric cancers after gastrectomy for synchronous multiple gastric cancers. World J Surg. 2010;34:296-302. PMID: 20012285.

9 Tanigawa N, Nomura E, Lee SW, Kaminishi M, Sugiyama M, Aikou T, et al. Current state of gastric stump carcinoma in Japan: based on the results of a nationwide survey. World J Surg. 2010;34:1540-7. PMID: 20182716.

10 Newman E, Brennan MF, Hochwald SN, Harrison LE, Karpeh MS, Jr. Gastric remnant carcinoma: just another proximal gastric cancer or a unique entity? Am J Surg. 1997;173:292-7. PMID: 9136783.

11 Sasako M, Maruyama K, Kinoshita T, Okabayashi K. Surgical treatment of carcinoma of the gastric stump. Br J Surg. 1991;78:822-4. PMID: 1873711.

12 Bozzetti F, Bonfanti G, Morabito A, Bufalino R, Menotti V, Andreola S, et al. A multifactorial approach for the prognosis of patients with carcinoma of the stomach after curative resection. Surg Gynecol Obstet. 1986;162:229-34. PMID: 3952614.

13 Maruyama K. The most important prognostic factors for gastric cancer patients. Scand J Gastroenterol. 1987;22:63-8. DOI: $10.3109 / 00365528709091021$.

14 Japanese classification of gastric carcinoma: 3rd English edition. Gastric Cancer. 2011;14:101-12. PMID: 21573743.

15 Tokunaga M, Sano T, Ohyama S, Hiki N, Fukunaga T, Yamada K, et al. Clinicopathological characteristics and survival difference between gastric stump carcinoma and primary upper third gastric cancer. J Gastrointest Surg. 2013;17:3138. PMID: 23233273.

16 Wang Y, Huang CM, Wang JB, Zheng CH, Li P, Xie JW, et al. Survival and surgical outcomes of cardiac cancer of the remnant stomach in comparison with primary cardiac cancer. World J Surg Oncol. 2014;12:21. PMID: 24468299.

17 Leivonen M, Nordling S, Haglund C. Does Helicobacter pylori in the gastric stump increase the cancer risk after certain reconstruction types? Anticancer Res. 1997;17:3893-6. PMID: 9427799.

18 Baba M, Konno H, Tanaka T, Kamiya K, Baba S, Sugimura $\mathrm{H}$, et al. Relationship of p53 and Helicobacter pylori to clinicopathological features of human remnant stomach cancer after gastric surgery for primary gastric cancer. Oncol Rep.
2001;8:831-4. PMID: 11410793.

19 Ikeguchi M, Saito H, Kondo A, Tsujitani S, Maeta M, Kaibara N. Mutated p53 protein expression and proliferative activity in advanced gastric cancer. Hepatogastroenterology. 1999;46:2648-53. PMID: 10522058.

20 Bani-Hani KE, Almasri NM, Khader YS, Sheyab FM, Karam HN. Combined evaluation of expressions of cyclin E and p53 proteins as prognostic factors for patients with gastric cancer. Clin Cancer Res. 2005;11:1447-53. PMID: 15746045.

21 Detsky AS, McLaughlin JR, Baker JP, Johnston N, Whittaker $\mathrm{S}$, Mendelson RA, et al. What is subjective global assessment of nutritional status? JPEN J Parenter Enteral Nutr. 1987;11:813. PMID: 3820522.

22 Vellas B, Villars H, Abellan G, Soto ME, Rolland Y, Guigoz Y, et al. Overview of the MNA--Its history and challenges. J Nutr Health Aging. 2006;10:456-63. PMID: 17183418.

23 Kondrup J, Rasmussen HH, Hamberg O, Stanga Z. Nutritional risk screening (NRS 2002): a new method based on an analysis of controlled clinical trials. Clin Nutr. 2003;22:32136. PMID: 12765673.

24 Onodera T, Goseki N, Kosaki G. [Prognostic nutritional index in gastrointestinal surgery of malnourished cancer patients]. Nihon Geka Gakkai Zasshi. 1984;85:1001-5. PMID: 6438478.

25 Nozoe T, Ninomiya M, Maeda T, Matsukuma A, Nakashima $\mathrm{H}$, Ezaki T. Prognostic nutritional index: a tool to predict the biological aggressiveness of gastric carcinoma. Surg Today. 2010;40:440-3. PMID: 20425547.

26 Ejaz A, Spolverato G, Kim Y, Poultsides GA, Fields RC, Bloomston $\mathrm{M}$, et al. Impact of body mass index on perioperative outcomes and survival after resection for gastric cancer. $\mathrm{J}$ Surg Res. 2015;195:74-82. PMID: 25619462.

27 Siewert JR, Bottcher K, Stein HJ, Roder JD. Relevant prognostic factors in gastric cancer: ten-year results of the German Gastric Cancer Study. Ann Surg. 1998;228:449-61. PMID: 9790335.

28 Kim JP, Kim YW, Yang HK, Noh DY. Significant prognostic factors by multivariate analysis of 3926 gastric cancer patients. World J Surg. 1994;18:872-877; discussion 877-8. PMID: 7846911.

29 Sakuramoto S, Sasako M, Yamaguchi T, Kinoshita T, Fujii M, Nashimoto A, et al. Adjuvant chemotherapy for gastric cancer with S-1, an oral fluoropyrimidine. N Engl J Med. 2007;357:1810-20. PMID: 17978289. 